\title{
Cidades globais no contexto dos negócios internacionais: Do esquecimento à tendência na produção acadêmica
}

\author{
Paulo Kazuhiro Izumi, Cyro Augusto Pachicoski Couto e Mário Henrique Ogasavara ${ }^{1}$ \\ Escola Superior de Propaganda e Marketing, ESPM/SP, São Paulo/SP, Brasil
}

DETALHES DO ARTIGO
Histórico do artigo:
Recebido em 26 de março de 2016
Aceito em 01 de novembro de 2016
Disponível online: 31 de dezembro de 2016
Sistema de Revisão "Double Blind Review"
Editor científico:
Ilan Avrichir

Palavras-chaves:

Cidades globais

Pesquisa em negócios internacionais

Estudo bibliométrico

Multinacionais

\begin{abstract}
RESUMO
Este artigo investiga a literatura de negócios internacionais dos últimos quarenta anos a partir da incorporação do termo cidades globais como referencial na construção de novos aportes teóricos. Foi realizada uma pesquisa bibliométrica em duas etapas. Na primeira, foram identificadas as principais áreas de conhecimento e os estudos seminais abordando o conceito. A maior parte das pesquisas concentram-se na geografia econômica e nos estudos urbanos. Na segunda, foram organizadas as abordagens utilizadas especificamente em negócios internacionais nos principais periódicos da área. Os resultados demonstram que os estudos sobre cidades globais são ainda incipientes destacando-se, dentre as abordagens, a relação entre vantagens de localização e aspectos específicos da empresa multinacional. Existe crescente interesse no desenvolvimento de análises em nível subnacional representado pelas cidades globais, a partir de diferentes aspectos como a escolha do modo de entrada, o papel das empresas de serviços avançados, a importância das redes e o processo de transferência de conhecimento. A combinação entre as perspectivas da geografia econômica e de negócios internacionais, embora reivindicada há algum tempo por estudiosos de ambas as áreas, ainda carece de um corpo teórico e empírico mais robusto no que se refere as cidades como centros de atratividade de investimentos estrangeiros em mercados globais. Ao analisar a importância do fenômeno das cidades globais, o estudo contribui para melhor compreensão das estratégias contemporâneas de internacionalização das empresas multinacionais, ressaltando o potencial interdisciplinar entre as teorias de negócios internacionais e geografia econômica.
\end{abstract}

(C) 2016 Internext | ESPM. Todos os direitos reservados!

\section{Introdução}

O surgimento, evolução e configuração das cidades globais (global cities) como fenômeno contemporâneo têm sido muito discutidos no campo da geografia econômica e nos estudos urbanos (Goerzen, Asmussen \& Nielsen, 2013). Nos últimos anos, no entanto, a expressão cidade global tem aparecido na literatura de negócios internacionais ainda de maneira superficial em comparação com outras áreas de pesquisa (Nachum, 2003; Nachum \& Wymbs, 2005; 2007). Embora ainda incipientes, os estudos têm apontado caminhos promissores no desenvolvimento de pesquisas, sobretudo no que se refere às decisões de localização do investimento direto estrangeiro em nível subnacional (Chadee, Qiu \& Rose, 2003; Chan, Makino \& Isobe, 2010; Chen, Li \& Shapiro, 2012) e também no que concerne aos efeitos das características de regiões subnacionais sobre a performance de subsidiárias internacionais (Ma, Tong \& Fitza, 2013; Nguyen, Le \& Bryant, 2013).

Em outro sentido, a própria emergência em se buscar novos aportes teóricos, conforme diversos autores da área apontaram (Kostova, Roth \& Dacin, 2008; Johanson \& Vahlne, 2009; Contractor, 2007; Hennart, 2012; Cantwell, Dunning \& Lundan, 2010), coloca as cidades globais como tema de vanguarda na 
produção acadêmica de negócios internacionais.

A conjuntura contemporânea econômica e social promove na academia a discussão sobre cidades globais também como tendência, despertando a atenção de pesquisadores das mais diferentes áreas sobre sua abrangência (Sassen, 1991), impacto social (Korf, 1987), estrutural (Santos, 2002), político (Friedmann, 1986), econômico (Shachar, 1983) e, principalmente, sobre os efeitos globalizantes gerados no mundo. Neste ínterim, as empresas passaram a se destacar na formação, desenvolvimento e sustentação das cidades, ao assumirem o papel de investidoras no espaço urbano, o que exigiu uma série de condições para as tornarem globalmente competitivas (Mcfarland \& Mcconell, 2011). Em contrapartida, as cidades evoluíram, deixando de ser apenas uma representação geográfica, assumindo assim uma condição de polo centralizador e multifacetado, com atuações diversas (Clark, 1996).

Contudo, existem lacunas empíricas quando o foco de análise se dá ao nível de cidades, seja na literatura de negócios internacionais (Goerzen, Asmussen \& Nielsen, 2013; Somers, Belderbos \& Du, 2014; Nachum \& Wymbs, 2007) seja no que se refere a uma potencial abordagem interdisciplinar entre essa e as áreas de estratégia e geografia econômica (Beugelsdijk, Mccann \& Mudambi, 2010; Beugelsdijk, Mudambi, Anderson \& Zaheer, 2013).

Mesmo presente em diferentes teorias de negócios internacionais, os estudos que consideram a "localização" como fator-chave da internacionalização (Dunning, 1998; Dunning \& Pitelis, 2008; Rugmann, 2009; Buckley \& Casson, 2009; Cantwell, 2009; Johanson \& Vahlne, 2009) não dedicaram a devida atenção ao tema cidades como objeto de pesquisa e construção teórica, o que só ocorreu de maneira relevante a partir do ano de 2000 (Beugelsdijk \& Mudambi, 2013; Blanc-Brude, Cookson, Piesse \& Strange, 2014; Goerzen et. al., 2013; Nachum \& Wymbs, 2007).

Embora não seja novo na área acadêmica, o termo cidades globais ganhou destaque na mídia mundial que relaciona o fenômeno a diversos assuntos (turismo, gastronomia, entretenimento, mobilidade, qualidade de vida, esportes, etc.), associando-o a características locais (mão de obra qualificada, recursos naturais, mercado consumidor, infraestrutura, etc.) e também em função de classificações anuais de cidades publicadas por empresas de consultoria de gestão e empresas de pesquisa (Kearney, 2012; Mastercard, 2008; Gawc 2012).

A formação das cidades globais é decorrente da transformação dos grandes centros urbanos em lugares de referência na rede de investimentos e comércio internacional (Friedmann, 1986; Sassen, 1991). A visão funcionalista adotada pelos estudos urbanos propõe a existência de uma hierarquia global de cidades que se estabeleceu com o desenvolvimento da economia pós-industrial e em função da capacidade dessas cidades de controlar o capital na nova divisão internacional do trabalho (Beaverstock, Taylor \& Smith; 1999; Castells, 1999). Esses locais caracterizam-se pela forte presença de empresas multinacionais, investimento direto estrangeiro, serviços financeiros e outras corporações de serviços especializados (Abrahamson, 2004).

Diante disso, segue a questão que permeou este trabalho: de que forma o conceito de cidades globais tem sido utilizado nos estudos de negócios internacionais? O objetivo é compreender a evolução da pesquisa em cidades globais no âmbito da literatura de negócios internacionais. O estudo foi conduzido por meio de método bibliométrico e envolveu duas etapas. Na primeira, foram identificados os estudos seminais ao desenvolvimento do conceito e as áreas de conhecimento relacionadas.

Este levantamento permitiu mapear de forma mais ampla e consistente o campo intelectual disponível e identificar conteúdos pertinentes à questão de pesquisa. Em um segundo momento foram organizadas as abordagens específicas adotadas em negócios internacionais em periódicos de grande prestígio da área.

Os resultados indicaram que o tema cidade global, mesmo ocupando posição central em outras áreas, ainda tem recebido pouca atenção da pesquisa em negócios internacionais. Apesar disso, a análise apresenta o tema como importante tendência de estudos. Existe um interesse emergente, que suscita várias possibilidades de desenvolvimento empírico, entre as quais, podem ser citados: a análise dos modos de entrada das empresas multinacionais (EMNs) nas cidades globais, a presença e o papel das empresas produtoras de serviços avançados, o papel das redes nas cidades globais e as transferências de conhecimento entre sedes e subsidiárias, a relação 
entre a estratégia de localização nas cidades globais e a busca de inovação, entre outras perspectivas.

Este estudo contribui para o avanço do conhecimento em negócios internacionais das seguintes formas: primeiro, ao avaliar, por meio de indicadores de produção científica, a evolução do conceito de cidades globais como fenômeno relevante para a compreensão das estratégias contemporâneas de internacionalização de empresas multinacionais. Segundo, ao reforçar o potencial interdisciplinar existente entre as teorias e conhecimentos de negócios internacionais e as áreas de estudos urbanos e geografia econômica. Terceiro, ao evidenciar a necessidade de novos estudos cuja unidade de análise seja o nível subnacional e, em particular, o nível de cidades.

O artigo foi estruturado em quatro partes. Primeiro são apresentadas a evolução do conceito de cidades globais e suas diversas abordagens. $\mathrm{Na}$ segunda parte são descritos o método, o procedimento e a amostra da pesquisa. $\mathrm{Na}$ sequência, com base no levantamento dos principais autores de negócios internacionais que abordaram o conceito, são analisados os caminhos de pesquisa trilhados e as principais contribuições realizadas. Por último, são apresentadas as conclusões e a possibilidade de estudos futuros.

\section{Cidades globais e seus múltiplos sentidos}

A expressão cidade mundial ou cidade global, conforme as diferentes abordagens e autores, foi utilizada pela primeira vez por Patrick Geddes em 1915 e retomada por Peter Hall em 1966 com a obra "World Cities" (Ferreira, 2003; Gavinha, 2008). Para Geddes (1998), o conceito de cidades globais estava relacionado à capacidade de cidades tornarem-se megalópoles, centros políticos, econômicos e articuladoras de espaços. Em outro sentido, Hall (1966) buscou uma abordagem política, definindo cidades globais como lugares onde se encontram os mais poderosos governos nacionais e também autoridades internacionais. Nota-se que em seu trabalho a noção de "lugar", aparece com destaque, além de ter sido o primeiro autor a enfatizar a condição econômica das cidades como aspecto relevante.

Em trabalho posterior, Shachar (1983) direciona o conceito de cidade global para uma perspectiva econômica. Para o autor, as cidades mundiais são regiões urbanizadas em grande escala, centros básicos de controle e acumulação do capital em dimensão internacional. Seu estudo influenciou o trabalho de pesquisadores posteriores, como foi o caso de Friedman (1986), que reforçou o conceito de cidade global ao reinterpretar o seu papel econômico nas transformações conhecidas como globalização. A partir disso, outros autores desenvolveram suas pesquisas sobre o tema (Cohen, 1981; Korff, 1987; Tabb, 1990; King, 1990) proporcionando-Ihe diversos sentidos e várias linhas de pesquisa nas áreas de geografia (Phelps \& Ozawa, 2003), sociologia (Alderson \& Beckfield, 2004), antropologia (Low, 1996), relações internacionais (Brenner, 1998) e, mais recentemente, negócios internacionais.

No início dos anos 1990, Saskia Sassen revigorou o conceito de cidades globais a partir de sua obra "The global city: New York, London, Tokyo" ao incorporar as noções de dispersão espacial e integração global. Em sua essência, as cidades globais constituem centros de domínio e influência estratégicos em função do poder de comando econômico mundial, posição geográfica privilegiada, influência nacional e internacional, sediando grandes corporações transnacionais e empresas de serviços avançados (advanced producer services - APS) (Sassen, 1991; Taylor, Derudder, Faulconbridge, Hoyler \& Ni, 2014).

Com isso, houve uma mudança de paradigma nas intervenções urbanas a partir da década de 90 (Nobre, 2000) tendo como estratégia a renovação do sentido da cidade que, além de um espaço urbano, é um local de negócios, atendendo as necessidades tecnológicas atuais e as novas condições de competitividade mundial. Sassen (1991) incorpora essa discussão em seu trabalho caracterizando a cidade global pela sua importância espacial, econômica, expressão política, cultural e como centro nodal competitivo em âmbito global. A questão de base proposta pela autora é que as estruturas fundamentais para a condução da atividade econômica mundial estão necessariamente localizadas nas cidades, ou seja, lugares específicos cujos espaços, dinâmicas internas e estrutura social importam globalmente.

Conforme Sassen (1991, p.3-4),

"Além de sua longa história como centros de comércio e
atividades bancárias internacionais, essas cidades agora
funcionam em quatro novas formas: primeira, como
pontos de comando altamente concentrados na
organização da economia mundial; segunda, como
localizações-chave para empresas financeiras e de 
serviços especializados que substituíram a manufatura como o setor econômico líder; terceira, como locais de produção, incluindo a produção de inovação nesses importantes setores; e quarta, como mercados para os produtos e as inovações produzidas."

O destaque dado a este pensamento de Sassen, reproduz a essência do que será trabalhado em negócios internacionais. O conceito de cidade global, portanto, passa a incorporar as empresas multinacionais como agentes essenciais de sua constituição, agindo não apenas na esfera econômica, como também na social, ao assumir funções do Estado (Ferreira, 2007).

\section{Metodologia}

Para compreender a literatura existente, identificar a estrutura intelectual e fundamentar a revisão de literatura foi realizado um estudo exploratório, com abordagem quantitativa, por meio de levantamento bibliométrico definido como uma "técnica quantitativa e estatística de medição dos índices de produção e disseminação do conhecimento científico" (Araújo, 2006, p. 12). Para isso, foi utilizado o software Publish or Perish, do Google. Trata-se de um programa desenvolvido por Anne-Wil Harzing (2007) que utiliza o Google Acadêmico como base de dados científicos.

Ao realizar a pesquisa nesse programa, o pesquisador recupera uma lista das referências informações científicas, artigos, periódicos, livros e eventos - pertinentes à expressão de busca e gera estatísticas de produtividade e de impacto de autores e periódicos tais como o número de citações e o índice-h, ou h-index (Mugnaini \& Streh, 2008), um dos indicadores bibliométricos mais conhecidos na ciência da informação que possibilita verificar a visibilidade de autores e artigos científicos (Oliveira et. al., 2015). O h-index foi proposto pelo físico Jorge Hirsch em 2005 para mensurar ao mesmo tempo a produtividade e o impacto do trabalho de um pesquisador, com base em seus artigos mais citados. $\mathrm{O}$ h-index de um pesquisador é definido como o maior número "h" de artigos científicos desse pesquisador que têm pelo menos o mesmo número "h" de citações cada um. Dessa forma, um pesquisador com h-index 30 é aquele que publicou pelo menos 30 artigos científicos que foram citados em pelo menos 30 outros trabalhos (Marques, 2013).

O método adotado envolveu duas grandes etapas no que se refere aos procedimentos de seleção e filtragem.

Inicialmente, foi realizada a quantificação das publicações com a expressão-chave "global cities", no período de 1972 a 2016. Esta primeira etapa objetivou compor uma amostra abrangente a fim de averiguar a evolução dos trabalhos desenvolvidos e a representatividade geral do tema em diferentes áreas.

A busca por uma literatura mais ampla possibilitou mapear de forma ampla o território intelectual disponível a fim de identificar conteúdos mais consistentes com a questão de pesquisa. Recentes estudos abordando o tema cidades globais mencionam um desenvolvimento mais profícuo em outras disciplinas, em especial, na geografia econômica e estudos urbanos (Goerzen et. al., 2013; Mccann, 2011; Nachum \& Wymbs, 2007; Mehlsen, 2014).

Este levantamento inicial resultou em 34.200 registros de publicações em diversas áreas do conhecimento. A partir disso, foi feita a seleção dos documentos classificadas com índice-h, resultando em 168 publicações.

Verificada a grande dispersão de resultados em áreas de conhecimento distintas de negócios internacionais, foram escolhidas as palavras-chave que representaram os filtros para a seleção das obras mais direcionadas ao eixo de pesquisa dos negócios internacionais. Para isso, foram realizadas duas combinações de termos para filtrar a busca. Na primeira, combinando as expressões "global cities" com "international business", o resultado gerado foi de 2.260 registros. Constatou-se, no entanto, o mesmo padrão observado no levantamento geral, ou seja, uma grande dispersão de resultados em áreas distintas de negócios internacionais. Assim, foi feita a segunda busca, combinando os termos global cities com multinational enterprise, no qual foram verificados 342 registros. Estes resultados se mostraram mais alinhados com o foco deste estudo e, a partir dele, foram feitas as seleções e recortes para a segunda etapa da análise bibliométrica, centrada na identificação das abordagens do assunto no contexto dos negócios internacionais.

Dos 342 registros encontrados, foram selecionados 9 artigos publicados em revistas científicas de forte prestígio das áreas de gestão (Strategic Management Journal, Journal of International Business e Asia Pacific Journal of 
Management), negócios internacionais e gestão internacional (Journal of International Business Studies e European Journal of International Management), geografia econômica (Economic Geography), informetria (Journal of Infometrics) e 2 artigos apresentados em conferências (Conference on location decisions of multinational enterprises: markets, cities or clusters?, Copenhagen e Druid Academic Conference), totalizando 11 trabalhos.

As duas etapas descritas possibilitaram, no conjunto, selecionar as obras mais relevantes para a elaboração da revisão bibliográfica apresentada neste artigo.

\section{Resultados}

A busca inicial pela expressão "Global Cities" na base de dados do Google Acadêmico, resultou em 34.200 obras. Observa-se pela amostra representada na tabela 1, que as duas obras mais citadas pertencem à mesma autora: Sassen (1991), com 10.568 citações e Sassen (1994), com 3.832 citações. A terceira obra mais citada é a de Friedmann (1986), com 2.664 citações. Tratam-se de autores e respectivas obras considerados seminais, que evoluíram da tradição funcional dos estudos urbanos. Esta abordagem enfatizou a interconectividade e o papel econômico das cidades globais como parte de um sistema maior, dos processos contemporâneos de globalização, diferentemente da tradição demográfica, mais focada nas implicações humanas e ecológicas de grandes concentrações populacionais, atualmente representada pelo estudo das megacidades (Beaverstock, Taylor \& Smith, 1999; Goerzen et al., 2013).

Das 168 publicações com produtividade e impacto realizou-se a contagem de revistas que mais publicaram artigos referenciando a expressão global cities em qualquer parte de seu conteúdo. Observase pela tabela 2 que 16 revistas publicaram no mínimo dois artigos no período considerado, destacando-se a Urban Studies, uma das principais revistas internacionais na área de estudos urbanos e planejamento, com 16 artigos publicados. Todas as demais revistas publicaram 1 artigo cada uma.

Tab 1

As 20 obras mais citadas

\begin{tabular}{|c|c|c|c|c|}
\hline Ordem & Citações & Autores & Título & Ano \\
\hline 1 & 10568 & Sassen, S. & $\begin{array}{l}\text { The global city: New York, London, Tokyo. } \\
\text { Princeton, NJ: Princeton University Press. }\end{array}$ & 1991 \\
\hline 2 & 3832 & Sassen, S. & $\begin{array}{l}\text { Cities in a world economy. Thousand Oaks, CA: } \\
\text { Pine Forge/Sage. }\end{array}$ & 1994 \\
\hline 3 & 2664 & Friedmann, J. & $\begin{array}{l}\text { The world city hypothesis. Development and } \\
\text { Change, v. } 17\end{array}$ & 1986 \\
\hline 4 & 1940 & Newman, P \& Kenworthy, J. & Sustainability and cities: overcoming automobile dependence. Island Press. & 1999 \\
\hline 5 & 1689 & Scott, A. J. & $\begin{array}{l}\text { Global city-regions: Trends, theory, policy. Oxford: } \\
\text { Oxford University Press. }\end{array}$ & 2002 \\
\hline 6 & 1448 & Kearney, M. & $\begin{array}{l}\text { The local and the global: The anthropology of globalization and transnationalism. } \\
\text { Annual review of anthropology. }\end{array}$ & 1995 \\
\hline 7 & 1443 & Scott, A. J. & $\begin{array}{l}\text { The cultural economy of cities: essays on the geography of image-producing } \\
\text { industries. Sage Publications. }\end{array}$ & 2000 \\
\hline 8 & 1424 & Harvey, D. & Spaces of global capitalism. Verso. & 2006 \\
\hline 9 & 1318 & Smith, N. & New globalism, new urbanism: gentrification as global urban strategy. Antipode. & 2002 \\
\hline 10 & 1301 & Soja, E. & $\begin{array}{l}\text { The stimulus of a little confusion: a contemporary comparison of Amsterdam and } \\
\text { Los Angeles. In: After Modernism - global restructuring and the Changing } \\
\text { Boundaries of City Life }\end{array}$ & 1992 \\
\hline 11 & 1085 & Brenner, N. & $\begin{array}{l}\text { Globalisation as reterritorialisation: the re-scaling of urban governance in the } \\
\text { European Union. Urban Studies. }\end{array}$ & 1999 \\
\hline 12 & 1042 & Cervero, R. & The transit metropolis: a global inquiry. Island Press. & 1998 \\
\hline 13 & 1019 & $\begin{array}{l}\text { Beaverstock, J. V., Smith, R. V. } \\
\text { \& Taylor, P. J. }\end{array}$ & A roster of world cities. Cities v. 16 . & 1999 \\
\hline 14 & 960 & Campbell, S. & $\begin{array}{l}\text { Green cities, growing cities, just cities?: Urban planning and the contradictions of } \\
\text { sustainable development. Journal of the American Planning Association. v. } 62 \text {. }\end{array}$ & 1996 \\
\hline 15 & 927 & Knox, P. \& Taylor, P. & World cities in a world-system. Cambridge University Press. & 1995 \\
\hline 16 & 916 & $\begin{array}{l}\text { Dicken, P., Kelly, P. F., Olds, K. } \\
\text { \& Yeung, H. }\end{array}$ & $\begin{array}{l}\text { Chains and networks, territories and scales: towards a relational framework for } \\
\text { analysing the global economy. Global networks. v. } 2 \text {. }\end{array}$ & 2001 \\
\hline 17 & 890 & Pacione, M. & Urban geography: A global perspective. Routledge. & 2009 \\
\hline 18 & 883 & $\begin{array}{l}\text { Featherstone, M., Lash, S. \& } \\
\text { Robertson, R. }\end{array}$ & Global modernities. Sage publications. & 1995 \\
\hline 19 & 869 & Smith, M. P. \& Guarnizo, L. E. & The locations of transnationalism. In: Transnationalism from below. Sixth Printing. & 1998 \\
\hline 20 & 860 & Steger, M. B. & Globalization. The Encyclopedia of Political Thought. & 2010 \\
\hline
\end{tabular}

Fonte: Elaborado pelos autores. 
Tab. 2

Revistas que publicaram no mínimo dois artigos (com índice $H$ )

\begin{tabular}{clcl}
\hline Ordem & Revista & Publicações & Grupo de áreas do conhecimento \\
\hline 1 & Urban Studies & 16 & Estudos urbanos e planejamento \\
2 & International Journal of Urban and Regional Research & 4 & Economia regional e urbana \\
3 & Progress in Human Geography & 4 & Geografia \\
4 & Annual Review of Anthropology & 3 & Antropologia e Demografia \\
5 & Journal of Ethnic and Migration Studies & 3 & Antropologia e Demografia \\
6 & World cities in a World System & 3 & Geografia econômica \\
7 & American Behavioral Scientist & 2 & Ciências sociais \\
8 & Cities & 2 & Estudos urbanos e planejamento \\
9 & Environment and Urbanization & 3 & Estudos urbanos e planejamento \\
10 & Geoforum & 2 & Geografia \\
11 & Global Environmental Politics & 2 & Estudos ambientais \\
12 & Journal of the American Planning Association & 2 & Estudos urbanos e planejamento \\
13 & Public Culture & 2 & Estudos culturais \\
14 & Regional Studies & 2 & Economia regional e urbana \\
15 & Review of International Political Economy & 2 & Economia política internacional \\
16 & Transactions of the Institute of British Geographers & 2 & Geografia \\
\hline
\end{tabular}

Fonte: Elaborado pelos autores.

Das 168 publicações com h-index, 113 referem-se exclusivamente a artigos e 55 a livros e outras informações científicas. Conforme pode se constatar pela tabela 3, a produção de artigos apresenta-se intensamente concentrada nos grupos de estudos urbanos, economia regional e planejamento (47\%), geografia e geografia econômica (22\%), seguida por estudos ambientais (7\%) e antropologia e demografia (7\%). A área de gestão estratégica (strategic management) cujo corpo de conhecimentos se situa mais proximamente ao campo de estudos de negócios internacionais possui apenas 1 produção (1\%).

Tab. 3

Publicações de artigos por grupo de áreas de conhecimento

\begin{tabular}{lcc}
\hline Grupo de áreas do conhecimento & F & $\%$ \\
\hline Estudos urbanos, economia regional e planej. & 53 & 47 \\
Geografia e geografia econômica & 25 & 22 \\
Estudos ambientais & 8 & 7 \\
Antropologia e Demografia & 8 & 7 \\
Sociologia & 6 & 5 \\
Inovação tecnologia e pesquisa & 3 & 3 \\
Estudos interdisciplinares & 3 & 3 \\
Estudos culturais & 2 & 2 \\
Economia política internacional & 2 & 2 \\
Ciências sociais & 2 & 2 \\
Gestão estratégica & 1 & 1 \\
\hline Total & 113 & 100 \\
\hline
\end{tabular}

Fonte: Elaborado pelos autores.
4.1 Abordagens de cidades globais no contexto de negócios internacionais

Os resultados da busca possibilitaram ratificar a preocupação existente na literatura quanto à produção ainda incipiente de estudos em negócios internacionais tendo por contexto as cidades globais. Por outro lado, os documentos identificados, embora ainda pouco representativos em termos quantitativos, permitiram delinear as principais abordagens de cidades globais e sua relação com as teorias utilizadas na literatura de negócios internacionais e possíveis caminhos para pesquisas futuras.

Observa-se pela tabela 4 que, dentre os 11 artigos sobre cidades globais relacionados a negócios internacionais, o estudo de Nachum foi o mais citado, com 170 citações, seguido pelo artigo de Yeung, com 96 citações. Os autores Nachum, Goerzen e Belderbos possuem mais de um artigo abordando o tema no contexto de negócios internacionais. O artigo de Goerzen et al. (2013) recebeu 28 citações. Todos os artigos foram publicados a partir de 2002, evidenciando que somente nos últimos 13 anos, o tema cidades globais tem despertado interesse mais concreto por parte dos estudiosos de negócios internacionais.

A tabela 5 apresenta as principais abordagens teóricas utilizadas na literatura de negócios internacionais. Dentre elas, destaca-se fortemente a teoria das vantagens de localização, a mais frequente nos estudos de empresas multinacionais envolvendo 
Tab. 4

Publicações com abordagens do tema cidades globais em negócios internacionais

\begin{tabular}{|c|c|c|c|c|c|}
\hline Rank & Cit. & Autores & Título do artigo / trabalho & Ano & Publicação \\
\hline 1 & 96 & Yeung, $\mathrm{H}$. & $\begin{array}{l}\text { Entrepreneurship in international business: An } \\
\text { institutional perspective }\end{array}$ & 2002 & $\begin{array}{l}\text { Asia Pacific Journal of } \\
\text { Management }\end{array}$ \\
\hline 2 & 30 & Nachum, L.. e Wimbs, C. & $\begin{array}{l}\text { Firm-specific attributes and MNE location choices: } \\
\text { financial and professional service FDI to New York and } \\
\text { London }\end{array}$ & 2002 & $\begin{array}{l}\text { ESRC Centre for Business } \\
\text { Research }\end{array}$ \\
\hline 3 & 170 & Nachum, L.. & $\begin{array}{l}\text { Liability of foreignness in global competition? Financial } \\
\text { service affiliates in the city of London. }\end{array}$ & 2003 & Strategic Management Journal \\
\hline 4 & 56 & Nachum, L. e Wymbs, C. & $\begin{array}{l}\text { Product differentiation, external economies and MNE } \\
\text { location choices: M\&As in global cities }\end{array}$ & 2005 & $\begin{array}{l}\text { Journal of International } \\
\text { Business Studies }\end{array}$ \\
\hline 5 & 28 & $\begin{array}{l}\text { Goerzen, A.; Asmussen, C. } \\
\text { e Nielsen, B. }\end{array}$ & $\begin{array}{l}\text { Global cities and multinational enterprise location } \\
\text { strategy }\end{array}$ & 2013 & $\begin{array}{l}\text { Journal of International } \\
\text { Business Studies }\end{array}$ \\
\hline 6 & 5 & $\begin{array}{l}\text { Taylor, P.; Derudder, J.; } \\
\text { Faulconbridge, J.; Hoyler, } \\
\text { M. e Ni, P. }\end{array}$ & $\begin{array}{l}\text { Advanced producer service firms as strategic networks, } \\
\text { global cities as strategic places }\end{array}$ & 2014 & Economic Geography \\
\hline 7 & 1 & $\begin{array}{l}\text { Belderbos, R.; Du, S.; e } \\
\text { Somers, D. }\end{array}$ & $\begin{array}{l}\text { Global Cities as Innovation Hubs: The Location of R\&D } \\
\text { investments by Multinational Firms }\end{array}$ & 2015 & DRUID Academy Conference \\
\hline 8 & 0 & $\begin{array}{l}\text { Belderbos, R.; Du, H.; e } \\
\text { Goerzen, A. }\end{array}$ & $\begin{array}{l}\text { Global city connectivity and the establishment of MNC } \\
\text { regional headquarters }\end{array}$ & 2015 & $\begin{array}{l}\text { Conference: Location Decisions } \\
\text { of Multinational Enterprises: } \\
\text { Market, Cities or Clusters? }\end{array}$ \\
\hline 9 & 2 & Csomós, G.; Tóth, G. & $\begin{array}{l}\text { Exploring the position of cities in global corporate } \\
\text { research and development: a bibliometric analysis by } \\
\text { two different geographical approaches. }\end{array}$ & 2016 & Journal of Informetrics \\
\hline 10 & 1 & $\begin{array}{l}\text { Blevins, D. P., Moschieri, } \\
\text { C., Pinkham, B. C. e } \\
\text { Ragozzino, R. }\end{array}$ & $\begin{array}{l}\text { Institutional changes within the European Union: How } \\
\text { global cities } \\
\text { and regional integration affect MNE entry decisions }\end{array}$ & 2016 & Journal of World Business \\
\hline 11 & 0 & Mehlsen, K.; Wernicke, G. & Global Cities and the Liability of Foreignness & 2016 & $\begin{array}{l}\text { European Journal of } \\
\text { International Management. }\end{array}$ \\
\hline
\end{tabular}

Fonte: Elaborado pelos autores.

cidades globais. Essa base teórica pode ser verificada nos estudos que relacionam as decisões de localização com as vantagens específicas da firma, no artigo de Nachum e Wymbs (2002); com o modo de entrada, no artigo de Nachum e Wymbs (2005); com as desvantagens de operar em mercados estrangeiros (liability of foreigness), nos artigos de Goerzen et al., (2013) e de Mehlsen e Wernicke (2016); com os investimentos de P\&D, no artigo de Belderbos, Du e Somers (2015); com as redes de conectividade entre cidades, no artigo de Beldebos, Du e Goerzen (2015).

Com efeito, o conceito de localização é uma áreachave tanto para os estudos de negócios internacionais quanto para a pesquisa em geografia econômica (Goerzen et al., 2013). Na literatura tradicional de negócios internacionais, as vantagens de localização do investimento direto estrangeiro são consideradas vantagens específicas do país (countryspecific advantages) que podem se originar tanto de instituições locais formais ou informais, quanto de recursos, capacidades e características de mercados locais (Dunning \& Lundan, 2008). O paradigma eclético ou modelo OLI (Dunning \& Lundan, 2008) postula que as empresas buscam se internacionalizar em função de três tipos de vantagens conjuntas: vantagens de posse ou propriedade (ownership), que são específicas da firma (firm specific advantages FSA), vantagens de localização (location), que são específicas do país (country specific advantages location) e internalização (internalisation).

Percebe-se que, embora fatores determinantes das escolhas de localização do investimento direto estrangeiro ocupem lugar central nos estudos de negócios internacionais (Beugelsdijk \& Mudambi, 2013; Dunning, 1998; Eden \& Lenway, 2001; Flores \& Aguilera, 2007), ao elegerem o nível país como foco de análise, esses estudos carecem de uma diferenciação mais precisa das nuances de características locais como explicação da distribuição da atividade econômica de multinacionais. Esta abordagem em nível subnacional e, especificamente no nível das cidades globais, é mais frequente em estudos de geografia econômica e de economia regional (Beugelsdijk et al., 2010; Crescenzi et al., 2015; Mccann \& Mudambi, 2005).

Ainda fundamentado no escopo teórico das vantagens competitivas, verifica-se pela tabela 5 a utilização do importante conceito de desvantagem de ser estrangeiro, ou seja, os custos adicionais e dificuldades de operar em mercados fora de seu país de origem (liability of foreignnes -LOF) que a empresa 
Tab. 5

Abordagens teóricas e abordagens de cidades globais

\begin{tabular}{ll} 
Autores & Título do artigo / trabalho \\
\hline Yeung, H. (2002) & Entrepreneurship in international \\
& business: An institutional \\
& perspective. Asia Pacific Journal of \\
& Management. v. 19, 2002.
\end{tabular}

Abordagens Teóricas Institucionalismo.

areg

Abordagens de cidades globais
Empreendedores internacionais precisam de
recursos financeiros de fora dos seus países e/ou
regiões de origem. É importante garantir o consentimento e reconhecimento por financistas globais, constituídos por banqueiros, gestores de fundos e corretoras. As principais cidades globais abrigam os financistas globais.

$\begin{array}{ll}\text { Nachum, L. \& } & \text { Firm-specific attributes and MNE } \\ \text { Wimbs, C. } & \text { location choices: financial and } \\ \text { (2002) } & \text { professional service FDI to New } \\ & \text { York and London. ESRC Centre for } \\ & \text { Business Research. University of } \\ & \text { Cambridge. Working Paper n. 223 }\end{array}$
2002.

Nachum, L. Liability of foreignness in global
(2003) competition? Financial service affiliates in the city of London. Strategic Management Journal. v. 24, 2003.

Vantagens de localização
de MNEs.

Características específicas de cidades globais afetam a decisão de localização de MNES. As subsidiárias precisam ser capazes de adquirir conhecimento do mercado em primeira mão, o que explica a localização em clusters centrais nessas cidades.

Liability of Foreigness LOF e Vantagem competitiva de MNEs (Firm Specific Advantage - FSA)

As características distintivas das cidades globais pode originar fontes de vantagens competitivas, afetando de maneira diferente a existência e a força da responsabilidade de ser estrangeiro LOF. Empresas de serviços financeiros estrangeiros que operam na cidade de Londres não sofrem a LOF na medida sugerida pela teoria.

Nachum, L. \& Product differentiation, external Wymbs, C. economies and MNE location (2005) choices: M\&As in global cities. Journal of International Business Studies. v. 36, 2005.

Vantagens de localização e modo de entrada via Investimento Direto Estrangeiro - FDI.

A diferenciação do produto combinada à vantagens de localização são estratégicas para a MNE. Escolhas de localização não são determinadas por vantagens de localizações mas por fatores específicos da firma. Fusões e aquisições em cidades globais podem ter características próprias.

Goerzen, A.; Global cities and multinational enterprise location strategy. Asmussen, C. \& Nielsen, B. (2013) Journal of International Business Studies. v. 44, 2013. Vantagens de localização, paradigma eclético - OLI e Liability of Foreigness LOF.

\section{As características distintivas das cidades globais -}

interconexão global, cosmopolitismo, e

abundância de serviços avançados - APS ajudam as empresas multinacionais a superar custos de LOF. As empresas multinacionais têm forte propensão a se localizarem dentro de cidades globais.

Taylor, P.; Derudder, J.; Faulconbridge, J.; Hoyler, M. \& NI, P. (2014) Belderbos, R.; Global Cities as Innovation Hubs: D. (2015) by Multinational Firms. DRUID Academy Conference. Jan., 15-17, 2014.

Belderbos, R.; Global city connectivity and the Du, H. \& Goerzen, A. (2015) establishment of MNC regional headquarters. Conference: Location Decisions of Multinational Enterprises: Market, Cities or Clusters? May, 12, 2015

Mehlsen, K. \& Wernicke, G. (2016). Global cities and liability of foreignness. European Journal of International Management. 2016.

Blevins, D. P.; Moshchieri, C.; Pinkham, B. C. \& Ragozzino, R. (2016)

Csomós, G. \& Tóth, G. (2016).

Redes de relacionamento Cidades globais são lugares estratégicos para e cidades como centro de empresas de serviços avançados - APS. Por sua empresas de serviços avançados - APS. Vantagens de localização de Investimento em P\&D, redes de inovação e clusters tecnológicos.

\section{Conectividade de} cidades, redes corporativas e vantagens de localização.

Vantagens de localização, liability of foreigness LOF e distância institucional.

\section{Mudanças institucionais} como determinante de modo de entrada.

Posicionamento de subsidiárias orientadas por P\&D na organização da MNE. vez, as APS são empresas chave que funcionam como redes estratégicas das quais as cidades globais precisam ser parte. Cidades globais são redes (hub) de inovação tecnológica que favorecem a localização de investimentos em P\&D de MNEs.

\section{A posição da cidade dentro de redes globais (a força da conectividade) tornam a MNE mais propensa a localizar suas sedes regionais (RHQs) nessas cidades globais e essa relação é mais pronunciada nas indústrias intensivas em conhecimento.}

\section{Empresas multinacionais têm forte propensão} para se localizar nas cidades globais inlfuenciadas pela distância institucional (LOF) e características industriais de P\&D. Cidades globais apresentam capacidade para reduzir a LOF sofrida por MNEs. Cidades globais impactam decisões de modo de entrada. As mudanças institucionais na Europa, a adesão à UE e as cidades globais configuram as opções de governança das MNEs.

\section{Cidades globais são, ao mesmo tempo, os} principais pontos de controle de P\&D (abordagem da cidade de origem) e locais de atividades internacionais de P\&D (abordagem da cidade de acolhimento)
Institutional changes within the European Union: How global cities and regional integration affect MNE entry decisions. Journal of World Business. 2016

Exploring the position of cities in global corporate research and development: a bibliometric analysis by two different geographical approaches. Journal of Informetrics. 2016.
Palavras-chave

Transnational

entrepreneurship;

Institutional perspective;

Actor networks; Business

systems; International

business.

\section{Foreign Acquisitions,}

Location Advantages,

Clusters, Global

Cities, Financial and

professional service industries

Financial services; City of

London; Liability of

foreigness; global

competition.

Product differentiation; Location decisions; Global cities; Foreign acquisitions; Financial and professional service industries.

Global cities; Liability of foreignness; Foreign direct investment; Alliances and Joint ventures; Location strategy;

Internationalization

Advanced producer services; Global cities; London, New York; Strategic places; World city network.

Multinational Firms, R\&D investment, global cities, connectivity.

Global cities; Regiona headquarters; Networks; Location choice.

Global cities; liability of foreignness; multinational enterprises; institutional distance; location choices. Institutional distance; location choices.

Global city Multinational company Corporate research and development Scientific article 
multinacional enfrenta diante das empresas locais do país estrangeiro (Zaheer, 1995).

A relação entre cidades globais e a liability of foreigness foi inicialmente sugerida por Nachum (2003) ao propor que características distintivas das cidades globais originam fontes de vantagens competitivas capazes de afetar diretamente a existência e a força da desvantagem de ser estrangeiro. Suas conclusões mostraram que, no contexto particular da cidade global de Londres, as empresas estrangeiras superaram os custos adicionais associados à sua operação estrangeira, apresentando vantagens e desempenho superiores às empresas locais.

Posteriormente, Goerzen et. al. (2013), verificaram que as empresas multinacionais são atraídas por cidades globais que fornecem vantagens locacionais especificas que ajudam a abrandar os efeitos negativos da LOF. A conexão internacional, a aglomeração de empresas de serviços avançados e o ambiente cosmopolita servem para aliviar os custos associados à incerteza, discriminação e complexidade de fazer negócios em um ambiente estrangeiro. A atratividade das cidades globais decorre desses seus atributos únicos e não do seu tamanho populacional. Também demonstraram que as empresas multinacionais exploradoras de competência e criadoras de competências (Cantwell \& Mudambi, 2005) seguem padrões espaciais bastante distintos em suas atividades: enquanto as primeiras tendem a se aglomerar em cidades globais, as últimas, mais valiosas para o desenvolvimento econômico local, tendem a se concentrar em regiões metropolitanas ou periféricas menos centrais (Goerzen et. al., 2013).

De maneira similar, Mehlsen e Wernicke (2016) verificaram que as empresas multinacionais têm maior propensão a se localizar em cidades globais do que em áreas metropolitanas ou periféricas. Essas escolhas de localização são afetadas pela distância institucional e pelas características industriais. Seus resultados também suportam empiricamente o argumento de que a localização em uma cidade global reduz a LOF sofrida pelas empresas multinacionais, além de que essas cidades desempenham um papel central no processo de globalização.

Outra abordagem observada em artigo constante da tabela 5 focaliza o modo de entrada do investimento direto estrangeiro por meio de fusões e aquisições em cidades globais. Segundo as teorias de internacionalização, as decisões de modo de entrada envolvem a escolha do nível de propriedade do capital (joint venture ou subsidária integral), a forma de estabelecimento da subsidiária (aquisição ou criação de uma nova empresa - investimento greenfield) e o tipo de atividade (produção ou distribuição) (Dias, Rocha \& Silva; 2014). O artigo de Nachum e Wymbs (2005) mostra que as fusões e aquisições em cidades globais podem ter características próprias, sobretudo, em se tratando de empresas de serviços financeiros que experimentaram grande expansão, especialmente em razão da internacionalização da propriedade do capital. Conclusões acerca de particularidades do investimento direto estrangeiro em cidades globais não podem ser generalizadas para diferentes áreas geográficas. Além disso, eles identificaram que, muitas vezes nas cidades globais, operam sedes internacionais de empresas locais e também sedes nacionais de empresas estrangeiras. Ou seja, as aquisições nas cidades globais implicam mais do que apenas ter a presença do escritório local, uma vez que o transbordamento de conhecimento (knowldege spillovers) proporciona às multinacionais acesso a centros de controle e conhecimento (Nachum \& Wymbs, 2005).

Yeung (2002) utiliza a perspectiva institucional para analisar o empreendedorismo internacional, um campo que, juntamente com a área da estratégia e de outras relacionadas a negócios vem ampliando seu interesse nas questões de localização e geografia econômica (Beugelsdijk, Mccann \& Mudambi, 2010; Mccann \& Mudambi, 2005). Em negócios internacionais, os estudos sobre efeitos de aspectos institucionais focam dois pontos de vista: "o impacto do ambiente institucional do país de origem sobre as decisões de modo de entrada e o impacto do ambiente institucional do país hospedeiro sobre essas decisões" (Rocha \& Ávila, 2015). As diferenças entre o ambiente institucional do país de origem e o do país hospedeiro foram explicadas pelo conceito de distância institucional (Kostova, 1999). Segundo esse conceito, o sucesso de transferências das práticas de uma empresa para suas subsidiárias será afetado pelo grau de semelhança ou diferença entre o ambiente institucional dos países de origem e acolhimento (Kostova \& Zaeer, 1999).

Para Yeung (2002) as relações institucionais que se estabelecem entre países de origem e acolhimento podem ser definidas pelas redes sociais e de negócios que incorporam empresários, estruturas político- 
econômicas e práticas organizacionais e culturais dominantes nos países de origem e de acolhimento. Empreendedores internacionais precisam de recursos financeiros de fora dos seus países e/ou regiões de origem. As relações institucionais são importantes para garantir o consentimento e reconhecimento por financiadores globais, constituídos por bancos, gestores de fundos e corretoras que possuem suas bases operacionais nas principais cidades globais que servem como centros de comando de seus investimentos globais (Yeung, 2002; Sassen, 1991).

O artigo de Blevins, Moschieri, Pinkham e Ragozzino (2016), por sua vez, focaliza as mudanças institucionais ocorridas durante o período de integração regional na União Europeia, que resultou em um ambiente empresarial unificado, e as vantagens locacionais de cidades globais como determinantes do modo de entrada de empresas multinacionais. Seus resultados apontaram que mudanças institucionais na Europa tais como a adoção de uma moeda comum, diretrizes mais claras, um ambiente jurídico mais integrado, entre outros benefícios, parece modificar as preferências de modo de entrada - de aliança estratégica para aquisições , embora esse efeito tenda a diminuir ao longo do tempo. Multinacionais têm padrões de aquisição significativamente diferentes quando investem dentro da União Europeia ao invés de empresas-alvo situadas fora da União Europeia e fora de uma cidade global. As cidades globais se tornaram menos atraentes para a entrada das multinacionais à medida que a integração institucional progrediu, reduzindo a incerteza em locais da União Europeia menos cosmopolitas do que as cidades globais. Além disso, as cidades globais possuem instituições que, historicamente, facilitaram o intercâmbio e atraíram o investimento estrangeiro, sendo consideradas centros únicos de estabilidade institucional tornando-as acessíveis e atraentes ao investimento direto estrangeiro (Blevins et. al. 2016; Goerzen et al., 2013).

Outra abordagem importante para cidades globais é a de redes. Taylor et al. (2014), explora a noção de que as cidades globais são lugares estratégicos para empresas de serviços avançados, tais como serviços financeiros, publicitários, de arquitetura e jurídicos que, por sua vez, funcionam como redes estratégicas em função de suas atividades de prestação de serviços para grandes corporações transnacionais. Assim, da perspectiva das cidades, estas empresas operam como redes estratégicas, das quais as cidades precisam fazer parte, mas, da mesma forma, da perspectiva das empresas, estas cidades são consideradas lugares estratégicos, onde as empresas precisar estar (Taylor et al., 2014). Seu estudo mostra que as cidades de Nova York e Londres têm diferentes níveis de capacidade estratégica e isso está relacionado com a capacidade de inovação da primeira e o papel de consumidora global de serviços da segunda.

Belderbos, Du e Somers (2015) propõem que a posição de centros nodais ocupada pelas cidades globais dentro de redes internacionais de investimento fortalece a interconectividade corporativa tornando a empresa mais propensa a localizar suas sedes regionais nesses locais. Essa relação é mais pronunciada nas indústrias intensivas em conhecimento. As cidades globais também funcionam como centros de inovação em redes internacionais de P\&D atraindo esse tipo de investimento por parte das Multinacionais (Belderbos et al, 2015).

Esta constatação é corroborada por Csomós e Tóth (2016) que, por meio de estudo bibliométrico, demonstraram que as cidades de Tóquio, Nova York, Londres e Paris situam-se no topo da hierarquia de cidades globais, tanto pela localização de centros decisórios das mais poderosas multinacionais no mundo comandando as atividades globais de P\&D (abordagem da cidade de origem) quanto pela localização de subsidiárias orientadas por atividades de P\&D e de centros corporativos de pesquisa, para onde são conduzidas as atividades de pesquisa (abordagem da cidade de destino), além da presença de startups atuantes nas indústrias que mais rapidamente crescem tais como nanotecnologia, biotecnologia e tecnologia de informação.

\section{Considerações Finais}

Este trabalho mapeou o perfil e a evolução dos temas relacionados às cidades globais nos últimos 40 anos em diferentes áreas e com ênfase nos estudos de negócios internacionais. Desta forma, o tema cidades, de forma geral e, mais especificamente, cidades globais, mesmo ocupando posição central na pesquisa de áreas distintas como a geografia econômica e estudos urbanos, é bem recente e ainda têm recebido pouca atenção na literatura de negócios internacionais. Esta produção incipiente decorre, em grande parte, do tradicional enfoque 
dado ao nível nacional de análise da gestão estratégica, das operações e das próprias teorias de internacionalização.

Mesmo assim, a análise conduzida apresenta o tema como uma importante tendência de pesquisa, tanto pela importância das abordagens crescentemente adotadas nos estudos empíricos de negócios internacionais quanto pela relevância do assunto na pesquisa em geografia econômica e estudos urbanos e economia regional. O nível de desagregação geográfica e a concepção mais sofisticada de lugar e espaço (Mccan \& Mudambi, 2005) tradicionalmente adotados nesses estudos passam a ser considerados importantes e mais precisos para a compreensão das estratégias e operações de empresas multinacionais. Por outro lado, a literatura analisada denota um interesse emergente, suscitando diferentes possibilidades de abordagens em negócios internacionais ao considerar o nível subnacional. Este estudo, portanto, reforça a noção de que o potencial para ampliar a força de análise nas pesquisas sobre cidades globais reside na combinação entre as perspectivas da geografia econômica e de negócios internacionais. Embora a importância desta concepção interdisciplinar tenha sido demonstrada anteriormente (Buckley, Ghauri, 2004; Mccann \& Mudambi, 2005; Mccann, 2011), parece ser um caminho ainda mais promissor quando se trata da temática das cidades globais no contexto dos negócios internacionais, oferecendo um terreno amplo e bastante frutífero para o desenvolvimento de novas pesquisas.

Os recortes temáticos mais promissores tendo por contexto as especificidades das cidades globais dentre uma diversidade de direções possíveis incluem: a escolha de modos de entrada, a importância das empresas de serviços avançados (APS - Advanced producer services) no processo de internacionalização e nas decisões de localização, o papel das redes e as transferências de conhecimento entre sedes e subsidiárias, estratégias de inovação e conhecimento, o processo co-evolucionário entre a EMN e o desenvolvimento econômico de cidades, os efeitos do ambiente institucional sobre as estratégias de internacionalização.

Uma limitação do estudo diz respeito ao reduzido número de artigos abordando diretamente as cidades globais sob a perspectiva de negócios internacionais. Essa condição trouxe dificuldades para gerar um entendimento profundo sobre as implicações das especificidades do contexto de cidades globais sobre as teorias de negócios internacionais. O que parece ficar evidente pelas análises é que há uma compreensão crescente da importância dos trabalhos de geografia econômica para um melhor desenvolvimento da teoria de localização e do impacto das cidades e de variáveis espaciais sobre a estratégia e o desempenho de multinacionais sob a lente de negócios internacionais.

Este estudo contribui para a literatura ao avaliar, por meio de indicadores de produção científica, a evolução do conceito de cidades globais enquanto fenômeno relevante para a compreensão das estratégias contemporâneas de internacionalização de empresas multinacionais. Além disso, esta investigação reforça o potencial interdisciplinar existente entre as teorias de negócios internacionais e de geografia econômica, além de evidenciar a importância de produzir novos estudos tendo o nível de cidades como unidade de análise.

\section{Referências}

- Abrahamson, M. (2004). Global cities. New York: Oxford University Press, 2004.

- $\quad$ Alderson, A. S., Beckfield, J. (2004). Power and position in the world city system1. American Journal of Sociology, 109(4), 811-851.

- Araújo, C. A. (2006). Bibliometria: evolução história e questões atuais. Em Questão, Porto Alegre, 12(1), 11-32.

- Beaverstock, J. V, Smith, R. G. \& Taylor, P. J. (1999). A roster of world cities. Cities, 16(6), 445-458.

- Belderbos, R., Du, H. \& Goerzen, A. (2015). Global city connectivity and the establishment of MNC regional headquarters. Paper session 3: "Global cities and MNE Location Choices". Conference on Location Decisions of Multinational Enterprises: Market, Cities or Clusters? Kraks Fond, Institute for Urban Economic Research. Copenhagen. Tuesday, May 12.

- Belderbos; R., Du, H. \& Somers, D. (2014). Global Cities as Innovation Hubs: The Location of R\&D Investments by Multinational Firms. DRUID Academy. Aalborg, Denmark.

- Beugelsdijk, S., McCann, P., \& Mudambi, R. (2010). Introduction: Place, space and organization - economic geography and the multinational enterprise. Journal of Economic Geography, 10(4), 485-493.

- Beugelsdijk, S. \& Mudambi, R. (2013). MNEs as bordercrossing multi-location enterprises: The role of discontinuities in geographic space. Journal of International Business Studies, 44(5), 413-426. 
- Beugelsdijk, S., Mudambi, R., \& Andersson, U. (2013). The Multinational in Geographic Space: Special issue in Journal of International Business Studies, 44(5), 413546.

- Blanc-Brude, F., Cookson, G., Piesse, J., \& Strange, R. (2014). The FDI location decision: Distance and the effects of spatial dependence. International Business Review, 23(4), 797-810.

- Blevins, D. P., Moschieri, C., Pinkham, B. C., \& Ragozzino, R. (2016). Institutional changes within the European Union: How global cities and regional integration affect MNE entry decisions. Journal of World Business, 51(2), 319-330.

- Brenner, N. (1998). Global cities, glocal states: global city formation and state territorial restructuring in contemporary Europe. Review of International Political Economy, 5(1), 1-37.

- Brenner, N. (1999). Globalisation as reterritorialisation: the re-scaling of urban governance in the European Union. Urban studies, 36(3), 431-451.

- Brown, E., Derudder, B., Parnreiter, C., Pelupessy, W., Taylor, P. J., \& Witlox, F. (2010). World City Networks and Global Commodity Chains: towards a worldsystems' integration. Global Networks, 10(1), 12-34.

- Buckley, P. J., \& Casson, M. C. (2009). The internalisation theory of the multinational enterprise: A review of the progress of a research agenda after 30 years. Journal of International Business Studies, 40(9), 1563-1580.

- Campbell, S. (1996). Green cities, growing cities, just cities?: Urban planning and the contradictions of sustainable development. Journal of the American Planning Association, 62(3), 296-312.

- Cantwell, J. (2009). Location and the Multinational Enterprise. Journal of International Business Studies, 40(1), 35-41.

- Cantwell, J., Dunning, J. H., \& Lundan, S. M. (2010). An Evolutionary Approach to Understanding International Business Activity: The Co-evolution of MNEs and the Institutional Environment. Journal of International Business Studies. 41(4), 567-86.

- Cantwell, J., \& Mudambi, R. (2005). MNE competencecreating subsidiary mandates. Strategic Management Journal, 26(12), 1109-1128.

- Castells, M. (1999). A era da informação: economia, sociedade e cultura (Vol. 1). Paz e terra.

- Cervero, R. (1998). The Transit Metropolis: A Global Inquiry. Island Press, Washington, D. C.

- Chadee, D. D., Qiu, F., \& Rose, E. L. (2003). FDI location at the subnational level: a study of EJVs in China. Journal of Business Research, 56(10), 835-845.

- Chan, C. M., Makino, S., \& Isobe, T. (2010). Does subnational region matter? Foreign affiliate performance in the United States and China. Strategic Management Journal, 31(11), 1226-1243.

- Chen, V., Li, J., \& Shapiro, D. (2012). Sub-national market-supporting institutions and outward fdi: evidence of emerging-market MNEs into developed markets. In Academy of International Business Conference, Washington, DC.

- Clark, D. (1996). Urban World/Global City. London/New York, Routledge.

- Cohen, R. B. (1981). The New International Division of Labor, Multinational Corporations, and the Urban Hierarchy, Urbanisation and Urban Planning in Capitalist Society, New York, 3, 309-344.

- Contractor, F. J. (2007). Is international business good for companies? The evolutionary or multi-stage theory of internationalization vs. the transaction cost perspective. Management International Review, 47(3), 453-475.

- Crescenzi, R., Pietrobelli, C., \& Rabellotti, R. (2015). Location Strategies of Multinationals from Emerging Countries in the EU Regions. LEQS Paper, (93).

- Csomós, G., \& Tóth, G. (2016). Exploring the position of cities in global corporate research and development: a bibliometric analysis by two different geographical approaches. Journal of Informetrics, 10(2), 516-532.

- Derudder, B., Witlox, F., \& Taylor, P. J. (2007). US cities in the world city network: Comparing their positions using global origins and destinations of airline passengers. Urban Geography, 28(1), 74-91.

- Dias, A. C. A. E. M., da Rocha, A. M. C., \& da Silva, J. F. (2014). Modos de Entrada no Investimento Direto no Exterior: Um Estudo da Literatura Empírica/Foreign Direct Investment Entry Modes: A Study of the Empirical Literature. Revista de Administração Contemporânea, 18(4), 416.

- Dicken, P., Kelly, P. F., Olds, K., \& Yeung, H. (2001). Chains and networks, territories and scales: towards a relational framework for analysing the global economy. Global Networks, 1(2), 89-112.

- Dunning, J. H. (1998). Location and the multinational enterprise: a neglected factor? Journal Of International Business Studies, 29(1), 45-66.

- Dunning, J. H., \& Lundan, S. M. (2008). Institutions and the OLI paradigm of the multinational enterprise. Asia Pacific Journal of Management, 25(4), 573-593.

- Dunning, J. H., \& Lundan, S. M. (2010). The institutional origins of dynamic capabilities in multinational enterprises. Industrial and Corporate Change, 19(4), 1225-46.

- Dunning, J. H., \& Pitelis, C. N. (2008). Stephen Hymer's contribution to international business scholarship: an assessment and extension. Journal of International Business Studies, 39(1), 167-176.

- Eden, L., \& Lenway, S. (2001). Introduction to the Symposium. Journal of International Business Studies, 383-400.

- Featherstone, M., Lash, S., \& Robertson, R. (Eds.). (1995). Global Modernities (Vol. 36). Sage. 
- Ferreira, J. S. W. (2003). São Paulo: o mito da cidadeglobal. São Paulo: Fauusp.

- Flores, R. G., \& Aguilera, R. V. (2007). Globalization and location choice: an analysis of US multinational firms in 1980 and 2000. Journal of International Business Studies, 38(7), 1187-1210.

- Friedmann, J. (1986). The world city hypothesis. Development and change, 17(1), 69-83.

- GaWC (2012). The World According to GaWC 2012. Globalization and World Cities research network. Diponível em <http://www.lboro.ac.uk/gawc/world2012t.html>. Acesso em 07 de fevereiro de 2016.

- Gavinha, J. (2008). Veinte años de ciudades globales: ideas, mitos, y nuevas evidencias. Scripta Nova: Revista Electrónica de Geografía y Ciencias Sociales, (12), 2.

- Geddes, P. (1998). Cities in Evolution, 1915. London Williams \& Norgate.

- Goerzen, A., Asmussen, C. G., \& Nielsen, B. B. (2013). Global cities and multinational enterprise location strategy. Journal of International Business Studies, 44(5), 427-450.

- Harzing, A.W. Publish or Perish. Disponível em: <http://www.harzing.com/pop.htm>. 2007. Acesso em: 01 jul. 2015.

- Harvey, D. (2006). Spaces of global capitalism. Verso.

- Johanson, J., \& Vahlne, J. E. (2009). The Uppsala internationalization process model revisited: From liability of foreignness to liability of outsidership. Journal of International Business Studies, 40(9), 1411-1431.

- Kearney, A. T. (2012). Global cities index and emerging cities outlook. The Chicago Council.

- Kearney, M. (1995). The local and the global: The anthropology of globalization and transnationalism. Annual review of anthropology, 547-565.

- King, A. D. (1990). Global cities: post-imperialism and the internationalization of London. Taylor \& Francis.

- Knox, P., \& Taylor, P. (1995). World cities in a world system. Cambridge University Press.

- Korff, R. (1987). The world city hypothesis: a critique. Development and Change, 18(3), 483-493.

- Kostova, T. (1999). Transnational transfer of strategic organizational practices: A contextual perspective. Academy of Management Review, 24(2), 308-324.

- Kostova, T., Roth, K., \& Dacin, M. T. (2008). Institutional theory in the study of multinational corporations: A critique and new directions. Academy of Management Review, 33(4), 994-1006.

- Low, S. M. (1996). The anthropology of cities: Imagining and theorizing the city. Annual Review of Anthropology, 383-409.

- Ma, X., Tong, T. W., \& Fitza, M. (2013). How much does subnational region matter to foreign subsidiary performance? Evidence from Fortune Global 500 Corporations' investment in China. Journal of International Business Studies, 44(1), 66-87.

- Marques, F. (2013). Os limites do Índice H. Pesquisa Fapesp, 207, 35-39.

- MasterCard Worldwide (2008) Worldwide Centers of Commerce Index 2008, MasterCard Worldwide, New York

- McCann, P. (2010). International business and economic geography: knowledge, time and transactions costs. Journal of Economic Geography, 11, 309-317.

- McCann, P., \& Mudambi, R. (2005). Analytical differences in the economics of geography: the case of the multinational firm. Environment and Planning A, 37(10), 1857-1876.

- McFarland, C. K., \& McConnel, K. J. (2011). Strategies for Globally Competitive Cities: Local Roles in Foreign Direct Investment and International Trade. Center for Research \& Innovation.

- Mathews, J. A. (2006). Dragon multinationals: New players in 21st century globalization. Asia Pacific Journal of Management, 23(1), 5-27.

- Mehlsen, K., \& Wernicke, G. (2016). Global cities and liability of foreignness. European Journal of International Management, 10(1), 78-94.

- Mugnaini, R., \& Strehl, L. (2008). Recuperação e impacto da produção científica na era google: uma análise comparativa entre o google acadêmico e a web of science 10.5007/1518-2924.2008 v13nesp1p92. Encontros Bibli: Revista Eletrônica de Biblioteconomia e Ciência da Informação, 13(1), 92-105.

- Nachum, L. (2003). Liability of foreignness in global competition? Financial service affiliates in the city of London. Strategic Management Journal, 24(12), 1187 1208.

- Nachum, L. (2010). When is foreignness an asset or a liability? Explaining the performance differential between foreign and local firms. Journal of Management, 36(3), 714-739.

- Nachum, L., \& Wymbs, C. (2002). Firm-Specific Attributes and MNE Location Choices: Financial and Professional Service FDI To New York And London. ESRC Centre for Business Research.

- Nachum, L., \& Wymbs, C. (2005). Product differentiation, external economies and MNE location choices: M\&As in global cities. Journal of International Business Studies, 36(4), 415-434.

- Nachum, L., \& Wymbs, C. (2007). The location and performance of foreign affiliates in global cities. Regional aspects of multinationality and performance. Research in Global Strategic Management, 13, 221-257.

- Nguyen, T. V., Le, N. T., \& Bryant, S. E. (2013). Subnational institutions, firm strategies, and firm performance: A multilevel study of private manufacturing firms in Vietnam. Journal of World Business, 48(1), 68-76. 
- Newman, P., \& Kenworthy, J. (1999). Sustainability and cities: overcoming automobile dependence. Island Press.

- Oliveira, A. B., Rodrigues, R. S., Blattmann, U., \& Pinto, A. L. (2015). Comparação entre o Qualis/CAPES e os índices H e G: o caso do portal de periódicos UFSC. Informação \& Informação, 20(1), 70-91.

- Pacione, M. (2009). Urban geography: A global perspective. Routledge.

- Pereira, R. O., \& Derudder, B. (2010). The cities/servicesnexus: determinants of the location dynamics of advanced producer services firms in global cities. The Service Industries Journal, 30(12), 2063-2080.

- Pereira, R. O., \& Derudder, B. (2010). The cities/servicesnexus: determinants of the location dynamics of advanced producer services firms in global cities. The Service Industries Journal, 30(12), 2063-2080.

- Phelps, N. A., \& Ozawa, T. (2003). Contrasts in agglomeration: proto-industrial, industrial and postindustrial forms compared. Progress in Human Geography, 27(5), 583-604.

- Da Rocha, A., \& Ávila, H. D. A. (2015). Teoria institucional e modos de entrada de multinacionais de países emergentes. Revista de Administração de Empresas, 55(3), 246.

- Rugman, a. M. (2009). Theoretical aspects of MNEs from emerging economies. In: Ramamurti, R; Singh, J. Emerging multinationals in emerging markets. United Kingdom: Cambridge University.

- Sassen, S. (1991). The global city: New York, London, Tokyo. Princeton University Press.

- Sassen, S (1994). Cities in a world economy. Thousand Oaks, CA: Pine Forge/Sage.

- Scott, A. (2002). Global city-regions: trends, theory, policy. Oxford University Press.

- Scott, A. J. (2000). The cultural economy of cities: essays on the geography of image-producing industries. Sage.

- Shachar, A. (1983). A cidade mundial e sua articulação ao sistema econômico global. In Becker, B. et. al.
Abordagens Políticas da Espacialidade. Rio de Janeiro, UFRJ.

- Shen, W. (2010). Globalizing Shanghai. International Migration and the Global City. World Institute for Development Economics Research. Working Paper, 9.

- Smith, N. (2002). New globalism, new urbanism: gentrification as global urban strategy. Antipode, 34(3), 427-450.

- Smith, M. P., \& Guarnizo, L. E. (Eds.). (1998). Transnationalism from below (Vol. 6). Transaction Publishers.

- Soja, E. (1992). The stimulus of a little confusion: a contemporary comparison of Amsterdam and Los Angeles. After Modernism: Global Restructuring and the Changing Boundaries of City Life, New Brunswick, NJ: Transaction Publishers, 17-38.

- Steger, M. B. (2010). Globalization. John Wiley \& Sons, Ltd.

- Taylor, P. J. (2001). Specification of the world city network. Geographical Analysis, 33(2), 181-194.

- Taylor, P. J., \& Aranya, R. (2008). A global 'urban roller coaster'? Connectivity changes in the world city network, 2000-2004. Regional Studies, 42(1), 1-16.

- Taylor, P. J., Derudder, B., Faulconbridge, J., Hoyler, M., \& Ni, P. (2014). Advanced producer service firms as strategic networks, global cities as strategic places. Economic Geography, 90(3), 267-291.

- TABB, W. (1990). As cidades mundiais e a problemática urbana: os casos de Nova York e Tóquio. Reestruturação Urbana: Tendências e Desafios São Paulo, Nobel/IUPERJ, 183-191.

- Yeung, H. W. C. (2002). Entrepreneurship in international business: An institutional perspective. Asia Pacific Journal of Management, 19(1), 29-61.

- Zaheer, S. (1995). Overcoming the liability of foreignness. Academy of Management Journal, 38(2), 341-363.

\section{Sobre os autores}

- Paulo Kazuhiro Izumi é Doutorando em Administração do Programa de Mestrado e Doutorado em Gestão Internacional da Escola Superior de Propaganda e Marketing, ESPM/SP, Brasil. E-mail: pki@uol.com.br

- Cyro Augusto Pachicoski Couto é Doutorando em Administração do Programa de Mestrado e Doutorado em Gestão Internacional da Escola Superior de Propaganda e Marketing, ESPM/SP, Brasil. E-mail:cyrocouto@yahoo.com.br

- Mário Henrique Ogasavara é Doutor em Administração pela University of Tsukuba, Japão. Atualmente é coordenador e professor do Programa de Mestrado e Doutorado em Gestão Internacional da Escola Superior de Propaganda e Marketing, ESPM/SP, São Paulo/SP, Brasil. E-mail: <mario.ogasavara@espm.br 


\title{
Global cities in the context of international business: From oblivion to tendency in scientific production
}

\author{
Paulo Kazuhiro Izumi, Cyro Augusto Pachicoski Couto and Mário Henrique Ogasavara
}

Escola Superior de Propaganda e Marketing, ESPM/SP, São Paulo/SP, Brasil

ARTICLE DETAILS
Article history:
Received 26 March 2016
Accepted 01 November 2016
Available online in 30 December 2016
Double Blind Review System
Scientific Editor
Ilan Avrichir

Keywords:

Global cities

international business research

bibliometric study

multinationals

\begin{abstract}
This paper investigates the international business literature of the last forty years from the incorporation of the term global cities as a reference in the construction of new theoretical contributions. A bibliometric method was performed in two stages. In the first one, the main areas of knowledge and the seminal studies addressing the concept were identified. Most research focuses on economic geography and urban studies. In the second, the approaches used specifically in international business were organized in the main journals of the area. The results show that the studies on global cities are still incipient, highlighting, among the approaches, the relation between location advantages and specific aspects of the multinational company. In addition, there is a growing interest in the development of analyzes at the subnational level represented by global cities, from different aspects such as the choice of entry mode, the role of advanced service firms, the importance of networks and the process of knowledge transfer. The combination of the perspectives of economic geography and international business, although claimed by scholars from both areas, still lacks a more robust theoretical and empirical body in regard to cities as centers of attractiveness of foreign investment in global markets. In analyzing the importance of the phenomenon of global cities, this study contributes to a better understanding of the contemporary internationalization strategies of multinational companies, highlighting the interdisciplinary potential between international business theories and economic geography.
\end{abstract}

(C) 2016 Internext | ESPM. All rights reserved!

Para citar este artigo:

Izumi, P. K.; Couto, C. A. P. e Ogasavara, M. H. (2016) Cidades globais no contexto dos negócios internacionais: Do esquecimento à tendência na produção acadêmica. Internext - Revista Eletrônica de Negócios Internacionais, 11 (3), 49-63. DOI:10.18568/1980-4865.11349-63

Para acessar este artigo: http://dx.doi.org/10.18568/1980-4865.11349-63 